\title{
Study on Robust Optimization Design Method Based on Random Probability Test
}

\author{
Hongqing Liu \\ Hunan vocational college of modern logistics,Changsha, Hunan,410131
}

Keywords: Optimization, Design, Random probability test, Transfer function.

\begin{abstract}
Based on the stochastic optimization design method of TMD is proposed. Gegenbauer polynomial approximation method of stochastic structure is transformed into an equivalent deterministic order system based on $\mathrm{H}$, select the norm of the transfer function expanded order system as the target of optimization using genetic algorithm to optimize the parameters of TMD. In addition, the stochastic seismic response and dynamic reliability of the stochastic structure TMD system before and after the control are analyzed and compared with the Monte Carlo method. The simulation results show that the proposed optimization method TMD can effectively reduce the random structural response peak and variance, improve the structure of the seismic reliability, using the method of optimal design of TMD has good robustness.
\end{abstract}

\section{Introduction}

As an effective passive control device, tuned mass damper (TMD) has been widely used to reduce the vibration response of structures under wind, earthquake and other external excitation [1 $\sim 5]$. The optimal design of TMD involves the real estimation of structural dynamic parameters. The uncertainty of structural parameters will be caused by various uncertain factors in practical engineering. In view of this situation, it is more appropriate to regard some parameters of structure as random variables. Therefore, the randomness of the structural parameters should be taken into account when optimizing the parameters of the TMD system. Aiming at the randomness and uncertainty in practical engineering, an optimal design method of stochastic structure TMD is proposed. Based on the theory of orthogonal polynomials of random parameters are consistent with the random distribution for a structured lambda PDF were determined by the minimum mean square error of the equivalent expanded order system [10,11], in order to reduce the randomness of the structure of the uncertainty caused by the impact on the effect of TMD control, the expanded order system in response to the $\mathrm{H}$ norm of the transfer function for the optimization goal, using genetic algorithm to optimize the parameters of TMD. Finally, a numerical example is given by the probability density evolution theory (PDEM) [12, comparative analysis of the structure of TMD system random response and dynamic reliability of 13], and using Monte Carlo method (Monte Carlo simulation) to verify the validity of the TMD stochastic structure optimization design method.

\section{Random probability test}

\subsection{Probability existence}

Here is another method of estimating probability, that is, maximum likelihood estimation. Maximum likelihood estimation is a method of parameter estimation, which is used to estimate the parameters of the distribution function in the case of known probability distribution. And the parameter of the distribution function is exactly the probability to be estimated.

The maximum likelihood estimation of such a simple idea: if based on a set of experimental data has been obtained, the probability distribution is known, can be the probability of the occurrence of this group of test data expressed as function parameters.

Many of you must have fainted when you see the above. I'll give you a specific example (a 
mathematically rigorous example, but consistent with the idea). I came to a strange university entrance, want to know the college boys or girls, I squatted in front of the school gate number 100 students out of school, found that 20 of the 80 boys and girls, if I think this school students in each school this time probabilities are more or less. Then I will infer the boys. Because more boys are more likely to produce the results of my observations. Therefore, the core idea of the maximum likelihood estimation is: to know the results, but do not know where the overall situation, and then calculate the probability in the overall results in each possible, have known results under what conditions the maximum probability, we think this is the overall situation.

Or was it the test of the ten coins I threw on the top?. According to the optimal frequency estimation and maximum likelihood estimation, the $\mathrm{P}$ (positive probability) is estimated to be 0.6. But if someone raises an objection and says that my estimate might be wrong, $\mathrm{P}$ actually is 0.5 , and my six appearance is positive because it's just an accidental result. I need to find evidence against him, because can not do numerous tests, I can only give a higher credibility of the evidence, for example, I want to prove the possibility of at least $95 \%$ of the six positive is because $\mathrm{P}$ is not equal to 0.5 , that is to say, that if $\mathrm{P}$ is 0.5 , the possibility of this result is not occasional I more than $5 \%$ ( $5 \%$ as significant).

\subsection{Classical probability}

As I said, what I'm going to refute is that the actual probability of flipping the coin is 0.5 , and then I'm going to prove that if $\mathrm{P}$ is 0.5 , then the probability of getting the result is very small (the upper requirement is less than $5 \%$ ).

The front face value is 1 , and the back value is 0 . If $\mathrm{P}$ is 0.5 , the value of each coin is subject to a 0-1 distribution of $\mathrm{P}$ 0.5. According to the definition of expectation and variance, the expectation and variance of this distribution are respectively:

As mentioned above, we hope that the significant level is $5 \%$, so I need to find $x=z$, the probability density function from $-\mathrm{z}$ to $\mathrm{Z}$ integral is 0.95 , then $0.632 \mathrm{in}[-\mathrm{z}, \mathrm{z}]$, if so, I think I do wrong, at least I have no more than $95 \%$ of the master said $\mathrm{P}$ is not equal to 0.5 , and if 0.632 is no longer the range, I can honestly say that I have been proved in theory that I have more than $95 \%$ grasp, $\mathrm{P}$ is not 0.5 (in other words, if $\mathrm{P}$ is 0.5 , ten times six times behind the positive probability of less than $5 \%$ ).

The theoretical basis of frequency estimation is the law of large numbers. It is no exaggeration to say that the law of large numbers is the most important cornerstone of the modern theory of probability and statistics, almost all correct statistical methods are dependent on the law of large numbers correctly, so the law of large numbers by some person first law of probability theory.

\subsection{Maximum likelihood estimation}

The law of large numbers intuitively shows the fact that under the same conditions, with the increase of the number of random trials, the frequency is getting closer to probability. Note that the law of large numbers is a tendency towards an infinite approximation of the true probability when the $\mathrm{n}$ tends to infinity.

When it comes to probability, you like to take flip coins for example. Most of the time, it's easy to think that the probability of the coin on the back is $1 / 2$, but it's not that simple. This article will take the coin toss test as an example and run through the full text, and lead to a series of basic content of probability theory and mathematical statistics. This article deals with classical probability, axiomatic probability, two item distribution, normal distribution, maximum likelihood estimation and hypothesis test. The main purpose of this paper is to explain the probability of modern mathematics and the basic mathematical statistics method based on probability theory.

\subsection{Significance hypothesis}

First of all, we have to answer a basic question: why does probability exist?. In fact, this is not a mathematical problem, but a philosophical problem. The reason to discuss this issue, because any mathematical activities are carried out in a certain philosophy under the premise, if not clear philosophical premise, mathematical activities can be conducted (e.g. if in your philosophy of probability does not exist, it also discussed what probability ah).

There is a certain probability in philosophy under the premise, I do not want to use philosophical 
terms in simple terms, is to drag the text, you must first acknowledge things exist objectively, and through observation and practice are abstract summary. For example, we often talk about "height". Why do we think height exists? Because we were observed after long-term practice found that a person's body height in the short term does not appear substantial changes, so we can use a number of units to describe a person's body in a relatively stable period in height. This is the "height" as a universally recognized philosophical premise.

Similarly, people in the long life, found in the world there are some things that the results are unpredictable, such as throwing coins to get the front or back, but later, some people find that, although the single results are not expected, but if I keep throwing, throwing a lot of times, positive results accounted for all the cast the number of coins was stable, and the number of more and more close to a fixed value. In other words, the coin toss, the single result is unpredictable, but the results of many trials are generally in conformity with the law (the term is called statistical laws).

\section{Conclusion}

Based on the orthogonal polynomial expansion theory, this paper presents an optimal design method of stochastic structure TMD, which is more in line with engineering practice. The effectiveness of the proposed method is verified by a numerical example. Through the analysis of the full text, we can get the following main conclusions: (1) the parameter uncertainty of the actual structure has great influence on the structural response, and the randomness of the structural parameters should be taken into account in the rational design of the TMD. TMD optimization design method proposed in this paper, the optimization process does not depend on the specific external excitation, the optimization design can reduce the sensitivity of the TMD control effect for structures with random parameters, improves the robustness of the TMD control effect, before the king has important theory significance and engineering application; (2) the probability density distribution for multi peak the curve of structural response, do not obey the normal distribution, logarithmic distribution and other commonly used probability distributions, if the direct use of the commonly used probability distribution approximation, may cause large errors; (3) using the method proposed in this paper to optimize the design of the TMD model can effectively reduce the random displacement peak average, reduced discrete response, and improve the the stochastic structure dynamic reliability under earthquake excitation, which means that the method is effective.

\section{References}

[1]Cui Liu, Yanhu Li, Yingyue Zhang, Chuluo Yang, Hongbin Wu, Jingui Qin, and Yong Cao, Solution-Processed, Undoped, Deep-Blue Organic Light-Emitting Diodes Based on Starburst Oligofluorenes with a Planar Triphenylamine Core, Chemistry - A European Journal, 2012, 18(22), 6928-6934.

[2] Dalia Abdelhamid, Hulya Arslan, Yingyue Zhang, and Kathryn E. Uhrich, Role of Branching of Hydrophilic Domain on Physicochemical Properties of Amphiphilic Macromolecules, Polymer Chemistry, 2014, 5(4), 1457-1462.

[3]Dalia S. Abdelhamid, Yingyue Zhang, Daniel R. Lewis, Prabhas V. Moghe, William J. Welsh, and Kathryn E. Uhrich, Tartaric Acid-based Amphiphilic Macromolecules with Ether Linkages Exhibit Enhanced Repression of Oxidized Low Density Lipoprotein Uptake, Biomaterials, 2015, 53, 32-39.

[4] Yingyue Zhang, Jennifer W. Chan, Alysha Moretti, and Kathryn E. Uhrich, Designing Polymers with Sugar-based Advantages for Bioactive Delivery Applications, Journal of Controlled Release, 2015, 219, 355-368.

[5]Yingyue Zhang, Qi Li, William J. Welsh, Prabhas V. Moghe, and Kathryn E. Uhrich, Micellar and Structural Stability of Nanoscale Amphiphilic Polymers: Implications for Anti-atherosclerotic Bioactivity, Biomaterials, 2016, 84, 230-240. 